\title{
Infection Control Policies in the NICU: Time for a review?
}

\section{Kelly Welton, RRT-NPS}

It is every NICU manager's worst nightmare: That your NICU has to close down because of an outbreak or a high rate of infection with the same pathogen. But ask any RT or RN who works at more than one NICU: policies vary from facility to facility.

As a Respiratory Therapist, it seems like we get blamed for every infection, there is. In many hospitals, the RT workload is divided very differently from the way nurses do it. Not many nurses have a couple of patients in one unit and other patients in another unit. It is just not done that way. Depending on the hospital, many RT's are given half a patient load in one unit and half a load in another area. RT's may have several patients on the general floors, for example, and 2 babies on HFNC in the NICU because that is how the workload points add up. But as you're going to see in a minute, it does not make much sense to do that.

All who enter the NICU must do what first? Some NICUs require a 3 minute surgical scrub upon entry, and some NICUs only require handwashing with soap or use of an alcohol-based rub. Let's consider ALL the people that do enter the NICU. There are doctors, not just the neonatologists but specialists such as eye doctors and surgeons. If your hospital is a teaching hospital, there may be residents that rotate through, not just for neonatology but surgical and pediatric cardiology residents. Physical therapists and Occupational therapists work with the babies on feeding, swallowing, and developmental care. Hearing professionals come in to screen the babies. Imaging people come in to do x-rays and ECHOs. Although most nurses work in one unit, some nurses may float to other units. But when nurses float, they are usually there for the whole shift, not jumping back and forth between 2 units. How about parents, foster parents, siblings, and grandparents. What does your facility require to enter the NICU?

\section{"Although most nurses work in one unit,} some nurses may float to other units. But when nurses float, they are usually there for the whole shift, not jumping back and forth between 2 units. How about parents, foster parents, siblings, and grandparents. What does your facility require to enter the NICU?"

After a visitor or staff member has done the initial wash or scrub, this seems to be where the rules change for everyone. Although family members will only visit their child or children, these fragile babies with immature immune capabilities may need to be better protected from their own parents. If mom and dad are able to hold their baby, is that a risk to the baby? What about Mom or Dad's hair? Staff are made to pull our hair back, yet parents' hair can drag all over a baby and their warmer. Most adults are well-colonized nasally with several different bacteria and then allowed skinto-skin right under their nose. What should the true standard be?

In term neonates, skin infections due to staph aureus, both MRSA and MSSA are most commonly found on the umbilical stump and on the groin. NICU staff and parents are said to be staph aureus nasal carriers. Neonates can handle being colonized but not overloaded. In very low birthweight or less than 1500 gram infants, the gram-positive organisms cause $70 \%$ of the infections, the majority being with coagulase-negative staph, which affects the skin and mucous membranes. Gram-negative pathogens cause 20\% of infections: E Coli, Klebsiella, Pseudomonas, Enterobacter, and Serratia. The remaining $10 \%$ are fungal, usually candida Albicans. Infection is generally facilitated by multiple invasive procedures that very low birth weight infants undergo, such as UAC's, UVC's, other lines, endotracheal intubation, and nasogastric tubes. This is all from a study done by Dr. Brenda Tesini, who does a lot of infectious disease studies. Let us pause and think: if it is mostly invasive procedures causing these infections, where are pathogens coming from, and how are they being transmitted? Let's go back to the gram-positive pathogens. We know that gram-positive organisms are on our hands and in our nose. As for the gram negatives, E Coli generally comes from the gut, so for example, if somebody changes a diaper and does not wash their hands well afterward or does not remove their gloves and gel before going on to the next task. Klebsiella is usually found in urine and gets transferred the same way - a quick diaper change, no hand hygiene, then touching the baby again. Pseudomonas is typically from water as it likes warm and moist environments but can also be found in urine. Pseudomonas infections often get blamed on the humidifier or ventilator circuits, but there is yet to be a good explanation of how it got there. Enterobacter can come from a UTI and also from a perforated bowel. Serratia is generally passed from hand-to-hand contact. The candida or fungal infections can come from an overgrowth caused by steroids or antibiotics. By killing both the good and the bad bacteria, the candida overgrows and can be passed to other babies.

What are some other factors that might cause a virus or bacteria to be transmitted from staff to baby or parent to baby? We encourage kangaroo care for mom and dad for the baby to spend some skin-to-skin time. Because we know that many pathogens normally live on our skin, could that be a cause of hospital-acquired infection? Are the parents required to wipe down with some CHG bath cloth first? When the eye doctor comes in to examine the baby, does he or she have a mask on? They get right up there with the scope looking into the baby's eyes, and even if the baby is intubated, could there be a risk of passing colonized pathogens to the baby? Respiratory therapists document ETT placement every 2 hours, requiring a very close look at those tiny endotracheal tubes to see the ETT depth at the lip or the gum. Nurses must document NG tube placement, also requiring a close look. Is a mask required to do those tasks?

Upon reviewing the current Center for Disease Control recom-

NEONATOLOGY TODAY is interested in publishing manuscripts from Neonatologists, Fellows, NNPs and those involved in caring for neonates on case studies, research results, hospital news, meeting announcements, and other pertinent topics.

Please submit your manuscript to: LomaLindaPublishingCompany@gmail.com 
mendations for NICU's, the only thing that stood out was a recommendation to perform active surveillance testing for Staph Aureus and MRSA colonization in NICU patients when there is an increased incidence of infection or in an outbreak setting. Current precautions for most NICUs are contact isolation when a baby is known to have an infection. Otherwise, it is hand hygiene and standard precautions only. Standard precautions essentially boil down to "Do what you need to do to protect yourself and prevent the spread of infection." Hand washing and use gloves when you know you're going to be handling something dirty. Should there possibly be a new class of precautions such as NICU precautions? What would that look like?

\section{"If a baby does end up in contact isolation, there is often a flyer or poster on the door or curtain. Are the instructions clear for visitors and family?"}

If a baby does end up in contact isolation, there is often a flyer or poster on the door or curtain. Are the instructions clear for visitors and family? Contact isolation requires dedicated or disposable equipment and clean and disinfect equipment before use on another person. Do all visiting MDs, OTs, and imaging people follow this? Who is monitoring? Moreover, back to the initial entry scrub: Should it be mandatory every time they come in? For example, some people come in at the start of their shift, do a 3-minute surgical scrub, and wash or gel in between patients. They leave to go to lunch, they are on their phone, they're in the cafeteria, they're handling stuff, they're talking with friends, and they come back into the NICU and then hand wash. Should they not do another 3-minute scrub? What about personnel who come in and treat patients and then leave to go take care of other patients in another unit? Think PT, OT, even RT. This has been the basis for some confusion for some time. Should they do a 3-minute scrub also every time they come in the unit? What about the gowning procedure? For example, somebody who has a split load, a few patients on the adult floors, and 3 NICU babies. They start their day on the adult floors in their scrubs, then they come into the NICU, do a 3-minute scrub, and put a gown on. Does that adequately protect the babies from what is on their scrubs? Or, what if they do the opposite - what if that therapist starts out seeing the babies in the NICU and then goes up to the adult floor? If they come in and if it is mandated, they put on NICU issued scrubs and see their babies and wash in between, and then they put a gown on when they go up to the adult floors, the idea being to keep their scrubs clean and take that gown off when they come back to NICU. Assuming they have no isolation patients upon the adult floors, is that good enough? Furthermore, if they did, many hospitals will insist that if standard precautions are being followed, there is no risk. However, how many times, [yes raise your hand], have you gone in to see a patient, $\mathrm{Neo}$, peds, or adult, and on the first round, the patient is clean, and you follow standard precautions. And the next time you go back to see those patients, one of them is now in isolation -- suddenly, there is a yellow cart by the door and a big sign. You have already exposed other patients and been exposed yourself to whatever this now-isolation patient has. Is it that effective to wear a gown as a barrier to your scrubs, take care of nonNICU patients, and take that gown off and go back into the NICU?

VAP - According to a meta-analysis done by a group of doctors in Switzerland, "Ventilator-associated pneumonia (VAP) is defined as a nosocomial lower airway infection, i.e., pneumonia, in intubated patients with onset after 48 hours or more of invasive mechanical ventilation ${ }^{1}(1)$. VAP is usually caused by airway colonization by potential pathogens, which disseminate due to inadequate immune response of the newborn's immature immune system. Sources of airway colonization can be the patient's own flora, i.e., bacterial overgrowth in oral secretions, reflux, and aspiration of gastric fluid, or the patient's environment with its caretakers and equipment $(\underline{2}, \underline{3})$. It also states, "There is a high diagnostic inconsistency and a low reliability of interpretation of chest X-rays regarding VAP." Meaning, if a baby is initially intubated and the CXR looks OK, and then 48 hours later, the CXR looks worse, that would be a presumed VAP. Because of the cost of VAP, even though it is difficult to define, diagnose, and treat, all hospitals have implemented a VAP bundle of some sort, usually requiring elevated head of bed, 24 or 48-hour change of inline suction catheters, no use of saline to suction, no changing or breaking a ventilator circuit unless necessary.

Infection cost to hospitals. In 2009 and 2010, various studies were published that said the hospital-acquired infection could cost as much as $\$ 14,000$ to $\$ 18,000$ extra over what it would have been had the patient not gotten an HAl. However, that is from 2001 data. According to inflation, it is now 20 years later, so in today's dollars, that cost is estimated at $\$ 20,000$ per patient incident. Let us do some general math: if you have $200 \mathrm{NICU}$ admissions per year on average, and half of those babies are affected by an $\mathrm{HAl}$, at an extra $\$ 20,000$, that is $\$ 2$ million in unnecessary treatments and unnecessary charges. Had we just been able to prevent the $\mathrm{HAI}$, we could have avoided the risks of antibiotics, the extra ICU days, and the stress on the parents of an extended NICU stay. And just keep that number in your head for a minute because we are going to come back to that.

What are the risk factors for a hospital-acquired infection? The risk is inversely proportional to the baby's gestational age, in addition to the number of lines the baby will need and the number of other-human exposures the baby will have.

The question is, is it time for a new NICU infection control protocol? Have best practices been identified worldwide? Say what you will about Facebook; here is a poll that I did in one of the Respiratory Therapy groups asking what the current infection control practice in your NICU is? The most common response at the time was: RT only has patients in the NICU no matter how slow it is. In other words, no mixing of loads, no assigning some patients that are adults or peds, and then some patients in NICU. That got the most votes, and that is a good thing. The second most popular option was in the "other" category, but only three people left comments and explanations. One says, "our charge is the only NICU RT that would see other patients. Our staff providing direct care only have NICU babies". That is good. The second commenter said, "What we practice is not what I feel is best" [ mixed loads].

The third person says, "If we don't have full staffing, they short staff the NICU instead of making a split assignment." That does not seem fair, but it has been responsible for staffing and scheduling. It can become quite the balancing act, and it looks like this hospital has decided on no mixing of patient populations. The third most popular answer was: if an RT has a patient load outside of the NICU, the RT can wear a cover gown in the NICU. I read that as they wear whatever they wear outside of NICU and then put a cover gown on to protect the babies when they come in NICU. One more vote says an RT with a mixed load must change into NICU-issued scrubs and use a cover gown to see patients outside of NICU. There was no absolute consistency as far as, should a non-designated RT or other staff put a gown on to come into NICU? Or, should they put a gown on when leaving NICU after seeing babies see other patients?

If we are going to improve NICU infection control policies, what would be considered extreme versus necessary? What does the 
evidence say about NICU visitors of any kind? Should we wear masks if we will be getting close to the baby? Should everybody that comes into the NICU wear NICU only scrubs? What is the current best practice with cover gowns, bodysuits, and generally protecting the babies from the things we pick up and carry on our clothing? What if you come to work in the NICU, change into NICU scrubs, do the three-minute wash, and then you get a call to go down to the ER to help out with a 30 weeker that popped out by surprise. Do you put a cover gown on to protect your scrubs while you go down to see this 30 weeker? When you come back to NICU, should you change scrubs again? How well do we clean our stethoscopes in between patients? How well does the ophthalmologist clean his equipment between babies? Who wipes down equipment after use and gets equipment ready for the next patient?

VAP and HAI numbers in the NICU must be reviewed to make a difference. Random surveillance must be done. I have long fought to get hooks at the doorway of every baby's bed to try to get people with lab coats, jackets, hoodies, sweaters, etc. to hang it up outside of the baby's room so they can keep an eye on it and everything that's in their pockets. Would that be a sound infection control measure to add at your hospital? Remember, at $\$ 20,000$ now per incident, depending on the number of admissions you have, whether it is $\$ 1,000,000$ or $\$ 2$ million a year would it be worth it for the hospital to hire an extra RT or two to avoid mixing loads? Or have you dedicated NICU PTs and OTs? If you could show that hiring more staff would save the hospital money, would you speak up?

I teach my students always to make it a point for people to see them hand wash and then gel, see them cleaning their stethoscope between babies, see them put their hair up or a cap on, and then wash or gel, not the other way around. No one likes to get blamed for spreading infection, but perhaps it is time for a more effective global policy as a starting point.

\section{References:}

1. Goerens, A; Lehnick, D; Buttcher, $M$ et al: Neonatal Ventilator Associated Pneumonia: A Quality Improvement Initiative Focusing on Antimicrobial Stewardship Front Pediatr. 2018; 6: 262. Published online 2018 Sep 24. doi: 10.3389/ fped.2018.00262 PMCID: PMC6165906 PMID: 30320046

2. Cernada $M$, Aguar $M$, Brugada $M$, Gutiérrez A, López $J L$, Castell $M$, et al. . Ventilator-associated pneumonia in newborn infants diagnosed with an invasive bronchoalveolar lavage technique: a prospective observational study. Pediatr Crit Care Med. (2013) 14:55-61. 10.1097/ PCC.0b013e318253ca31

3. Hooven TA, Polin RA. Pneumonia. Semin Fetal Neonatal Med. (2017) 22:206-13. 10.1016/j.siny.2017.03.002

Disclosure Statement: The author has no relevant conflicts of interest to declare

Readers can also follow NEONATOLOGY TODAY via our Twitter Feed @NEOTODAY

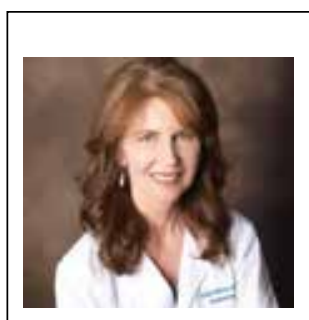

Corresponding Author

Kelly Welton, RRT-NPS

President,

Academy of Neonatal Care

La Quinta, California, United State

www.AcademyofNeonatalCare.org

Phone: 877-884-4587

Email: Educator@academyofneonatalcare.org
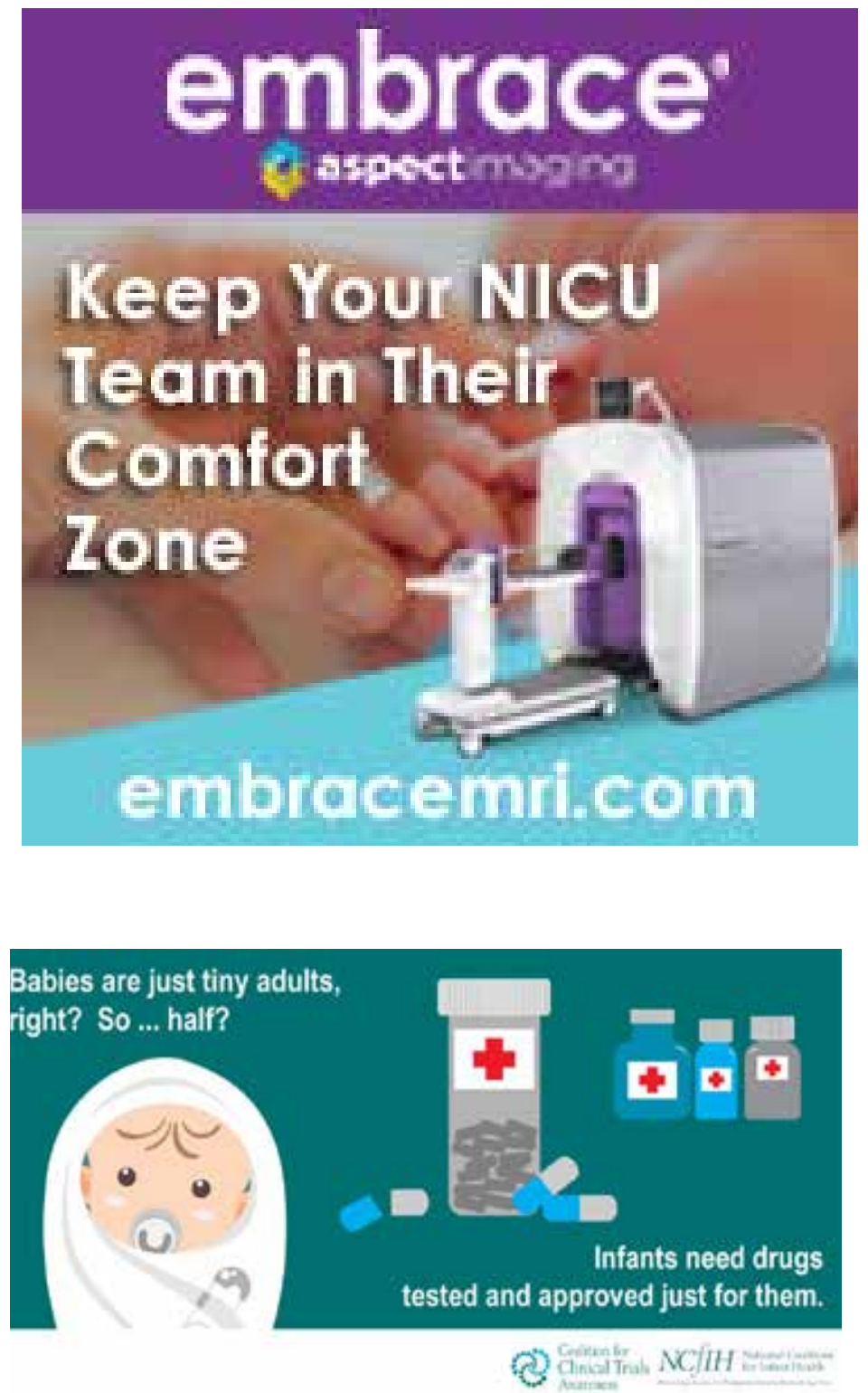